\title{
EFEITO DA DESIGUALDADE NO CRESCIMENTO ECONÔMICO DOS MUNICÍPIOS BRASILEIROS: UMA ANÁLISE COM BASE NO J ACKKNIFE MODEL AVERAGING
}

\begin{abstract}
Dércio Assis*
Fabricio Linhares *

Christiano Penna *

\section{RESUMO}

O presente artigo investiga a relação entre crescimento econômico e desigualdade de renda levando em consideração a incerteza com relação à especificação do modelo econométrico. Para evitar uma especificação inadequada, aqui faz se uso da técnica de ponderação de modelos proposta em Hansen e Racine (2012) conhecida por J ackknife Model Averaging. Esta técnica consiste em estimar todas as possíveis especificações de modelos com um amplo conjunto de variáveis e então calcular um modelo que é uma média ponderada de todos os outros estimados. A escolha dos fatores de ponderação é feita com base na minimização do critério de validação cruzada. Dado o elevado custo computacional, a análise foi feita levando-se em consideração três cenários. Apesar do esforço de pesquisa, nossos resultados sugerem que não há evidências de que a desigualdade de renda exerceu influência sobre a taxa de crescimento da economia brasileira no período de 2000 a 2009.
\end{abstract}

Palavras-chave: Crescimento Econômico, Desigualdade de renda e J ackknife Model Averaging.

\begin{abstract}
This paper investigates the relationship between economic growth and income inequality taking into account the uncertainty with respect to the econometric model specification. To avoid inappropriate specification, we use a model averaging technique proposed in Hansen and Racine (2012), that is known as Jackknife Model Averaging. This method consist of estimates all possible specifications of models with a wide range of variables and then calculate a model that is a weighted average of all other estimated. The choice of the weighting factors is based on minimizing the cross-validation criterion. Given the high computational cost, the analysis was made taking into consideration three scenarios. Despite the research effort, our results suggest that there is no evidence that income inequality exerted influence on the growth rate of the Brazilian economy in the period 2000-2009.
\end{abstract}

Keywords: Economic Growth, Income Inequality and Jackknife Model Averaging.

Códigos J EL: 040, 015, C10.

\footnotetext{
* Instituto de Pesquisa e Estratégia Econômica do Ceará - IPECE

- CAEN/UFC
} 


\section{INTRODUÇÃO}

A desigualdade de renda tem lugar cativo na agenda de pesquisa econômica, em especial na que trata de desenvolvimento e crescimento econômico. No caso brasileiro este tema é uma prioridade, pois nos destacamos de modo negativo entre as economias. O nível de renda per capita do Brasil não o qualifica como um país pobre no cenário internacional, já que, cerca de $64 \%$ dos países do mundo têm renda per capita inferior à brasileira1; contudo, apesar de ser relativamente rico, o Brasil é um país extremamente desigual (Barros, Henriques e Mendonça, 2000).

A relação entre crescimento econômico e desigualdade de renda começou a ser alicerçada na década de 90, a partir dos modelos endógenos de crescimento. Levadas por essa corrente, diversas teorias procuraram descrever quais seriam os efeitos da desigualdade de renda no crescimento econômico, e diversos estudos empíricos emergiram buscando corroborar ou refutar o que estas teorias prescreviam.

Várias abordagens empíricas foram realizadas na tentativa de estabelecer a direção do efeito da desigualdade de renda sobre o crescimento econômico. A metodologia mais comum empregada para testar a direção desse efeito tem como referencial as "Regressões de Solow Aumentadas", as quais ganharam evidencia nos trabalhos de Barro (1991), Mankiw, Romer e Weil (1992) e Barro e Sala-i-Martín (1992).

A análise aqui delineada concentra-se no caso particular em que se tem uma equação de regressão onde a taxa média de crescimento do PIB per capita é uma função do nível de desigualdade de renda no tempo inicial e de um conjunto de variáveis determinantes do crescimento. Regressões com essa estrutura foram propostas inicialmente no trabalho de Persson e Tabellini (1994), e replicadas nos trabalhos de Perotti (1996), e de Banerjee e Duflo (2003), por exemplo. Estas regressões sugenem que o sinal e a significância estatística do coeficiente da variável desigualdade definiriam a direção do seu efeito sobre o crescimento econômico.

Os estudos empíricos aplicados ao caso brasileiro seguem a estratégia de análise dos estudos cross-country citados anteriormente. Dentre estes estudos pode-se fazer referência aos trabalhos de Barreto, Neto e Tebaldi (2001), Castro e Pôrto J unior (2007), Amorim e Silva (2010) e Teyssier (2013).

Em termos empíricos - tanto para o caso brasileiro, quanto para o caso crosscountry - o que se observa é que, de um modo geral, não há um consenso entre a literatura que investiga o assunto. Existem vários trabalhos apontando para um efeito positivo [Li e Zou (1998), Forbes (2000)], outros tantos apontando para um efeito negativo [Alesina e Rodrick (1994), Person eTabellini (1994), Perroti (1996) Deninguer e Squire (1996), Barreto, Neto e Tebaldi (2001), Castro e Pôrto J unior (2007)], há trabalhos que apontam para efeitos diferenciados (Barro, 2000), assim como há trabalhos que não evidenciam qualquer efeito [Oliveira e Neri (2009), Amorim e Silva (2010) e Teyssier (2013)].

Lopez (2004) faz um levantamento detalhado de estudos cross-country retratando esta questão. Em suma, o que emerge da análise é que os resultados dependem basicamente da delimitação da amostra, do método de estimação e da definição do conjunto de variáveis determinantes do crescimento.

\footnotetext{
${ }^{1}$ Estatísticas recentes do Word Bank demonstram que o Brasil já apresenta renda per capita superior a $71 \%$ dos países do globo. Porém, está entre os $20 \%$ mais desiguais em distribuição de renda em ranking elaborado com base no coeficiente de Gini.
} 
Aqui se advoga que, ao se ter um amplo conjunto de variáveis a disposição do pesquisador, ${ }^{2}$ esta incongruência de resultados possivelmente decorre da incerteza na especificação do modelo. Mais especificamente, faz-se necessário ressaltar que as "Regressões de Solow Aumentadas" aparentemente apresentam um problema, pois não se sabe ao certo quais e quantas variáveis deveriam ser incluídas no modelo juntamente com o regressor de desigualdade.

Penna e Linhares (2015) argumentam que regressões de crescimento costumeiramente são feitas em dois estágios: primeiramente, um modelo é subjetivamente escolhido como "apropriado" e, no segundo estágio, são feitas inferências a partir deste modelo admitindo-se que o mesmo é o modelo estatístico que melhor se ajusta aos dados.

Essa estratégia empírica, entretanto, pode resultar num viés, pois se ignora a incerteza com relação aos demais modelos que poderiam ser propostos no primeiro estágio. Se o modelo subjetivo não for o mais adequado à estrutura dos dados, então a variância dos estimadores tende a ser subestimada. Isso traz problemas para os intervalos de confiança do modelo e, por conseguinte, permite inferências equivocadas a partir deste modelo.

A Ponderação de Modelos, ou Model Averaging, busca tratar a incerteza diante da especificação do modelo com base na ponderação de possíveis modelos alternativos, ou busca ponderar os melhores modelos indicados por algum critério. A ponderação de modelos pode ser feita com base em técnicas Frequentistas ou Bayesianas. ${ }^{3}$

O objetivo deste trabalho é contribuir para a literatura que investiga o efeito da desigualdade sobre o crescimento econômico levando-se em consideração a incerteza na especificação do modelo. A metodologia de estimação aqui empregada é o J ackknife Model Averaging (J MA), o qual faz uso de um esquema de ponderação ótima para os possíveis modelos construídos a partir de todas as variáveis explicativas disponíveis. ${ }^{4}$ Esta metodologia é aplicada a um banco de dados bastante amplo, com informações de 17 variáveis explicativas para 1486 municípios brasileiros.

Além desta introdução, este artigo divide-se em mais três seções. A seção que se segue faz uma revisão de literatura, onde são expostas as argumentações teóricas e as evidências empíricas sobre a relação entre crescimento econômico e desigualdade de renda. A terceira seção faz uma descrição da base de dados utilizada e da metodologia J ackknife Model Averaging, além de estipular cenários para análise. Na quarta seção são apresentados os resultados e as considerações finais do estudo.

\section{REVISÃO DA LITERATURA}

A literatura que investiga a relação entre desigualdade e crescimento pode ser dividida em dois grupos: um primeiro grupo seguiu a análise seminal de Kuznets (1955) - que sugere que a desigualdade de renda tende a se ampliar conforme a economia vai se desenvolvendo, atinge um ápice, e depois declina - e seu interesse

\footnotetext{
2 Durlauf e Quah (1999), por exemplo, listam um total de 87 variáveis "potencialmente explicativas" sugeridas na literatura. A atualização desta análise em Durlauf, J ohnson e Temple (2005) afirma que este número já havia subido para 145.

3 Ver Moral-Benito (2015) para um survey sobre o tema.

${ }^{4}$ Hansen e Racine (2012) o critério de seleção de pesos baseado no J ackknife, se comparada ao critério de Mallows, é mais apropriado para modelos lineares, pois permite que os erros aleatórios tenham, inclusive, variância heterocedástica, além de permitir que os modelos sejam não aninhados (ou nonnested).
} 
manteve-se focado no efeito do crescimento econômico na desigualdade de renda $(Y \rightarrow$ $D$ ); o segundo grupo focou-se na relação inversa entre estas variáveis, ou seja, abstevese a analisar o efeito da desigualdade no crescimento econômico $(D \rightarrow Y)$.

Uma análise conjunta destas duas vertentes tornaria este artigo demasiadamente extenso e fugiria do escopo que limita a análise. O ideal seria ter-se primeiro evidencias de causalidade entre estas variáveis para, posteriormente, investigar qual seria o efeito da variável explicativa na explicada. Mendes (2013), entretanto, aponta que os estudos econométricos realizados até então têm sido incapazes de demonstrar um claro padrão de relação causal entre a desigualdade e crescimento. Muitos problemas impõem limites a esses estudos: causalidade reversa, erros de medição, viés de amostra e a não linearidade são algumas dessas barreiras.

Com efeito, salienta-se aqui que este estudo se concentra apenas na ideia de que a desigualdade de renda afeta o crescimento econômico. A presente seção discute o arcabouço teórico apresentado na literatura cujo foco é esta relação, bem como busca detalhar alguns dos resultados já encontrados em trabalhos empíricos anteriores, tanto em âmbito internacional quanto nacional.

\subsection{Aspectos Teóricos}

Não existe ainda um consenso na literatura econômica sobre qual o impacto da desigualdade de renda sobre o crescimento econômico. Assim como há argumentos teóricos que apontam para uma relação negativa entre as duas variáveis, existem também explicações para uma provável relação positiva. Barro (2000) faz uma ótima revisão desses argumentos. A análise deste autor apresenta sete grupos de ideias distintas: i) $\mathrm{O}$ surgimento de atividades ilícitas; ii) $\mathrm{O}$ aumento nas despesas improdutivas; iii) A presença de maior carga tributária; iv) A criação de instabilidade político-institucional; v) Tamanho do mercado interno; vi) A restrição de crédito; e vii) Taxas de poupança.

Inicialmente pode-se inferir que em sociedades muito desiguais, maiores são os incentivos para que os indivíduos se engajem em atividades fora do mercado formal como crimes, drogas, contrabandos, etc. Isso além de configurar desperdício de capital humano, que poderia estar sendo alocado em alguma atividade produtiva, contribui para minorar a arrecadação tributária dos governos.

Por outro lado, a repressão a essas atividades acarreta dispêndio de recursos que poderiam ser usados em atividades produtivas. Muitas vezes, os governantes são obrigados a aumentar seus gastos no aparelhamento militar, construção e reformas de delegacias, criação de centros de detenção, entre outras atividades, que evidentemente são despesas relativamente improdutivas, no sentido de se ter um impacto relativamente pequeno na capacidade de geração de riqueza.

Questões relacionadas à decisão política dos eleitores majoritários também surgem como importante argumento sobre os efeitos nocivos da desigualdade sobre o crescimento econômico. Person e Tabellini (1994) e Alesina e Rodrick (1994), por exemplo, enfatizam esta questão. A ideia principal é que, diante de uma distribuição desigual de renda e riqueza, o eleitor mediano, desprovido de ativos e renda, desejaria ganhos no curto prazo através de uma redistribuição de renda do capital para o trabalho. Desta forma, através do processo político, programas partidários que anunciem a taxação do capital tenderiam a ser sancionados nas urnas, implicando numa elevação da carga tributária. Essa elevação dos tributos resultaria em menor acumulação de capital e, por conseguinte, numa menor taxa de crescimento da economia no futuro. 
Outro ponto a se observar é que os conflitos sociais decorrentes de uma forte desigualdade podem levar a uma maior instabilidade político-institucional gerando maiores incertezas no ambiente econômico, reduzindo assim os investimentos e a capacidade da economia de se ajustar a choques. Essa ideia é discutida nos trabalhos de Perotti (1996), Rodrick (1998) e Bourguignon (2000). Outra característica decorrente de elevados índices de desigualdade é a supressão do mercado consumidor, que certamente teria um efeito negativo nas vendas, na ampliação das plantas das fábricas e nos projetos de investimentos por parte das empresas, o que influenciaria negativamente o crescimento da atividade econômica.

Outro argumento, este levantado em Galor e Zeira (1993), refere-se à presença da restrição de crédito. Sabe-se que o processo de desenvolvimento econômico é caracterizado pela complementaridade entre o capital físico e capital humano, tal que o crescimento do estoque de capital requer um aumento subjacente do capital humano. Dessa forma, a restrição de crédito prejudicaria os indivíduos mais pobres a investir em capital humano, já que eles teriam maiores dificuldades em antecipar rendas futuras pela falta de garantias a esses empréstimos. Ademais, a falta de crédito também inibe o espírito empreendedor presente naqueles indivíduos mais carentes, o que dificulta novas gerações de negócios e renda nesse segmento da sociedade.

Por fim, há o argumento da acumulação de capitais, ou seja, da taxa de poupança da economia. Alguns economistas, dentre eles Denninger e Squire (1996), acreditam que as taxas de poupança individuais aumentam com o aumento do nível de renda; assim, a redistribuição de recursos dos indivíduos mais ricos para os mais pobres reduziria a taxa de poupança agregada da economia. Através deste canal, políticas de contenção de desigualdades tenderiam a diminuir o investimento. Com efeito, a redução das desigualdades implicaria num menor crescimento econômico, pelo menos durante a fase de aplicação da política de contenção.

\subsection{Estudos Empíricos}

A partir do início dos anos 90, com o advento das teorias do crescimento endógeno e a crescente disponibilidade de dados macroeconômicos, ressurge o interesse pelos fatores determinantes do crescimento econômico. Neste sentido, a desigualdade na distribuição de renda surgiu como um fator preponderante para explicar crescimento e, a partir daí, vários trabalhos empíricos foram realizados com o intuito de se investigar tal relação.

Em âmbito cross-country, pode-se destacar Alesina e Rodrick (1994), que utilizaram o Método dos Mínimos Quadrados Ordinários e uma amostra de países compreendendo o período de 1960 a 1985. Os autores regrediram a taxa média de crescimento do PIB per capita sobre os coeficientes de Gini da renda e da propriedade da terra, além do PIB per capita e percentual de educação primária (com ambas as variáveis sendo medidas no início do período). Neste estudo ficou evidenciado que tanto a desigualdade de renda quanto a de terra contribuem negativamente para o crescimento econômico.

Persson eTabellini (1994) regrediram a fração da renda apropriada pelo terceiro quintil, o PIB per capita inicial, o ensino primário e a participação política contra a taxa de média de crescimento, considerando um conjunto de países entre 1960 a 1985. Estes autores também concluem que desigualdade influência de modo negativo o crescimento.

Para o mesmo período do trabalho de Alesina e Rodrick (1994), Perotti (1996) estimou a taxa de crescimento do PIB per capita como função de um conjunto de 
variáveis: parcela da renda apropriada pelos terceiro e quarto quintis da distribuição de renda, PIB per capita de 1960, médias dos anos de estudo do ensino secundário da população masculina e feminina do ano de 1960, participação média de gastos do governo com segurança social e bem-estar com relação ao PIB para o período de 1970 a 1985, participação média de gastos do governo com educação e saúde em relação ao PIB no período de 1970 a 1978, taxa de urbanização, expectativa de vida, taxa de fertilidade, participação média do imposto de renda na renda pessoal no período de 1970 a 1988 e um conjunto de variáveis dummy. O autor conclui que desigualdade é prejudicial ao crescimento.

Com os avanços nesta linha de pesquisa, amplas bases de dados para países passaram a ser compiladas. Fazendo uso de uma destas bases, Deininger e Squire (1996) estimaram a relação entre crescimento e desigualdade, e concluíram que há uma forte relação negativa entre a desigualdade inicial na distribuição de terra e crescimento de longo prazo. Segundo os autores políticas que aumentassem o investimento agregado e facilitassem a aquisição de bens pelos pobres poderiam beneficiar duplamente o crescimento econômico e a redução da pobreza.

Li e Zou (1998) sugeriram estimar a relação entre crescimento-desigualdade através da técnica de efeitos fixos e efeitos aleatórios, desse modo, realizaram uma série de regressões, e, observaram que, ao regredirem a taxa de crescimento do PIB per capita sobre o índice de Gini e o PIB per capita no período inicial, as taxas de investimento, de crescimento populacional, de matricula do ensino primário e de urbanização, as exportações sobre PIB (como proxy para abertura comercial), e o desenvolvimento financeiro, encontravam que o efeito da desigualdadeno crescimento era significativo em vários casos, e positivo em todos eles.

Atentando para a questão de um possível viés associado ao efeito fixo resultante de estimações com dados em painéis, Forbes (2000) empregou o estimador do Método dos Momentos Generalizados, em primeira diferença, sugerido por Arellano e Bond (1991) e estimou o crescimento como uma função de desigualdade inicial, do PIB per capita, da educação masculina e feminina, do nível de investimento, e de variáveis dummy temporais e por país. Seus resultados demonstraram que, para prazos de 5 a 10 anos, a relação entre desigualdade de renda e crescimento é positiva.

Utilizando variáveis como índice de Gini, PIB per capita do período inicial, média dos anos de estudo da população masculina, consumo do governo, investimento como proporção do PIB, índice de democracia, inflação, taxa de fertilidade e taxa de crescimento em termos de comércio, Barro (2000), através do método dos mínimos quadrados em três estágios, apresentou evidências que efeitos negativos da desigualdade de renda sobre o crescimento se manifestam principalmente em países mais pobres, enquanto que países ricos apresentavam uma relação positiva entre essas variáveis. Seus resultados apontam que o crescimento tende a cair com uma maior desigualdade quando o PIB per capita está abaixo de aproximadamente US\$2000 e aumenta com a desigualdade quando o PIB per capita está acima de US\$ 2000.

Utilizando o banco de dados de Deininger e Squire (1996), Banerjee e Duflo (2003) sugeriram a existência de uma relação não linear entre desigualdade e crescimento. Através de métodos não paramétricos, os autores revelam que análises que assumem uma relação linear entre estas variáveis tem fraco suporte dos dados, e mostram que a relação entre desigualdade e crescimento seria condizente com o formato de um U invertido, sugerindo, assim, que alterações na desigualdade acarretariam em menor crescimento no futuro. 
Em se tratando de dados estaduais, ainda em âmbito internacional, Ortega-Diaz (2006) ressalta que a relação entre distribuição de renda e crescimento econômico depende de vários fatores, tais como imperfeições no mercado de crédito, risco moral e indivisibilidade no investimento. Com isso em vista, o autor constrói variáveis que buscam absorver tais questões e utiliza técnicas de estimação para um painel dinâmico que considera 32 estados do México. O autor encontra evidencias de que a desigualdade e crescimento são positivamente relacionados.

Oliveira e Neri (2009) fazem uso de um painel dinâmico estimado pelo método System-GMM e utilizam variáveis como Gini, PIB per capita, escolaridade masculina e feminina, além de uma proxy para distorção de mercado. Os autores trabalham com uma base de dados mais ampla do que a empregada pela literatura até então, além de propor especificações alternativas, e encontra evidências favorecendo a correlação positiva entre desigualdade e crescimento. Entretanto, ao utilizar um banco de dados mais restrito, obtém resultados defendendo uma relação negativa entre desigualdade e crescimento. Contudo, seus resultados demonstram que, tanto as evidências de uma relação positiva quanto de uma relação negativa, embora estatisticamente significativas, possuíam baixa significância econômica, dada a magnitude de seus coeficientes estimados.

Os resultados encontrados pela literatura com foco no caso brasileiro também sugerem a mesma contrariedade vista nos estudos cross-country. Barreto, Neto e Tebaldi (2001), analisando a região Nordeste encontraram uma relação negativa entre desigualdade e crescimento econômico. Castro e Pôrto Júnior (2007) utilizando o método proposto por Banerjee e Duflo (2003) encontraram uma relação negativa e, detectaram também, que variações na desigualdade, em qualquer direção, estariam associadas a uma menor taxa de crescimento no período seguinte.

Almeida e Guilhoto (2006), através do instrumental de matriz Insumo-Produto, investigam a contribuição dos diferentes setores da economia brasileira para o crescimento econômico e seus impactos sobre a distribuição de renda, e relatam que os setores que mais contribuem para o crescimento econômico diferem daqueles que melhoram a distribuição de renda, demonstrando, desse modo, uma possível relação negativa entre crescimento e desigualdade.

O estudo de Amorim e Silva (2010), que utilizou variáveis proxy's para capital físico e humano, além de um indicador de pobreza e o índice de Theil para captar desigualdade, verificou empiricamente o efeito da pobreza e da desigualdade de renda sobre a taxa de crescimento econômico das microrregiões de Minas Gerais entre os anos de 1980 e 2000, constatando que a relação entre desigualdade de renda e crescimento econômico não foi estatisticamente significativa, indicando que para as microrregiões mineiras, no período em análise, a distribuição de renda não afetaria o desempenho econômico.

Com base no trabalho de Marrero e Rodríguez (2012) realizado para os EUA, Teyssier (2013) parte do princípio de quea desigualdade de renda pode ser decomposta em desigualdade de oportunidades e desigualdade de esforço. Enquanto a primeira pode ser benéfica para o crescimento, a desigualdade de oportunidades serial prejudicial ao mesmo. O autor desenvolve variáveis proxy para os estados brasileiros capazes de assimilar tais efeitos e investiga qual destas variáveis teria um efeito dominante sobre a outra. Ao contrário do resultado encontrado para os EUA, o que se observa é que nem a desigualdade de esforço nem a desigualdade de oportunidades teriam um efeito significante sobre o crescimento, independentemente da especificação econométrica que se utilize. 


\section{METODOLOGIA}

A ideia central deste estudo é calcada numa regressão de crescimento Solow Aumentada, a qual pode ser descrita da seguinte forma:

$$
\Delta Y=\alpha+\beta X+\gamma Z+\varepsilon,
$$

onde $\Delta Y$ é um vetor $n \times 1$ contendo a taxa média de crescimento dos municípios entre 2000 e2009, $X$ éuma matriz $n \times k$ contendo variáveis relevantes que devem fazer parte de todas as estimações e, $Z$ é uma matriz $n \times k$ com variáveis de controle incertas, as quais podem ou não ser incluídas no modelo.

Como é discutido a seguir, tem-se ao nosso dispor um total de 17 variáveis explicativas. Deste modo, se forem estimados todos os modelos possíveis, sem fixação alguma de regressores, teríamos $2^{17}=131.072$ modelos a serem estimados. ${ }^{5}$ Este número é demasiadamente alto, impondo limitações computacionais que requerem que o exercício empírico aqui conduzido seja feito a partir de cenários.

O que se chama aqui de cenários refere-se ao número de regressores pertencentes às matrizes fixa, $X$, e variável, $Z$, descritas em (1). No caso da matriz $X$, fixou-se (i) a nossa variável de interesse, i.e. o Índice de Gini, e, como é de praxe em regressões de crescimento, fixou-se (ii) o PIB per capita para o período inicial e, (iii) a taxa de crescimento populacional mais depreciação. A opção por fixar essas duas últimas variáveis advém do fato de que as mesmas são prontamente sugeridas pelo modelo de Solow. Com efeito, a matriz $X$ é composta destas três variáveis, as quais são empregadas em todas as regressões.

Com relação à matriz de regressores variáveis, $Z$, o que se fez foi permitir que as variáveis disponíveis fossem combinadas de maneiras diversas: como não se sabe ao certo de que maneira tais variáveis devem realmente ser combinadas, a análise delineada a seguir envolveu combinações dos 3 regressores fixos (coletados na matriz $X$ anteriormente discutida) e estimou-se modelos com combinações de 4, 8 e 12 regressores auxiliares. ${ }^{6}$

\subsection{Dados}

Posto isso, torna-se relevante discutir em maiores detalhes o conjunto de dados aqui empregado. No presente trabalho optou-se por selecionar um grande número de variáveis as quais, intuitivamente, acredita-se ter forte relação com crescimento econômico. Foi feito também um levantamento dos regressores utilizados nos principais estudos sobre o tema. Ao todo foram coletadas 18 variáveis sugeridas pela literatura, todas elas amplamente disponibilizadas para o caso brasileiro ${ }^{7}$.

\footnotetext{
${ }^{5}$ Isso advém do conceito que todo espaço amostral finito possui $2^{\mathrm{n}}$ combinações prováveis.

${ }^{6}$ No caso do modelo com 4 regressores auxiliares, por exemplo, tem-se o intercepto mais três variáveis explicativas fixas (Gini, PIB per capita do período inicial e taxa de crescimento populacional mais depreciação) e quatro regressores variáveis, regressores estes que podem ser escolhidos do total de 14 . Foram estimados para este cenário $c_{4}^{14}=1001$ modelos, o que representa a combinação de 14 variáveis escolhidas 4 a 4 . No segundo cenário foram estimados $c_{8}^{14}=3003$ e no terceiro caso estimou-se $c_{12}^{14}=$ 91 modelos.

${ }^{7}$ Os dados foram obtidos no Censo Demográfico 2000 disponibilizado pelo IBGE, no banco de dados do Banco Central do Brasil e no Atlas do Desenvolvimento Humano desenvolvido pelo Programa das Nações Unidas para o Desenvolvimento (PNUD). Foram utilizadas informações dos municípios para os quais se tinham dados de todas as variáveis explicativas.
} 
Quadro 1- Descrição das Variáveis.

\begin{tabular}{|c|c|}
\hline & DESCRICÑ \\
\hline$\Delta \mathrm{Y}$ & Taxa de Crescimento do PIB per capita 2000 - 2009 \\
\hline \multirow{3}{*}{$\mathrm{X}$} & Coeficiente de Gini da renda domiciliar per capita 2000 \\
\hline & PIB per capita do ano 2000 em R\$ 2009 \\
\hline & Crescimento populacional $2000-2009$ \\
\hline \multirow{14}{*}{$\mathrm{Z}$} & Média das exportações sobre PIB entre 2003 e 2005 \\
\hline & PIB agrícola/ PIB total \\
\hline & Gastos Correntes do Governo / PIB \\
\hline & População Rural / População Total \\
\hline & Proporção de residência com água encanada \\
\hline & Proporção de residência com energia elétrica \\
\hline & Mortalidade infantil \\
\hline & Esperança de vida ao nascer \\
\hline & Proporção de Analfabetos \\
\hline & Média de anos de estudo \\
\hline & Proporção de Pobres \\
\hline & Distância da capital (Km) \\
\hline & Operações de crédito / PIB em 2000 \\
\hline & Depósitos / PIB em 2000 \\
\hline
\end{tabular}

Fonte: IBGE. PNUD. Elaborado pelos autores.

Conforme exposto, a variável dependente é a taxa média de crescimento do PIB per capita para o período de 2000 a 2009; as variáveis que serão mantidas fixas em todas as regressões, ou seja, as variáveis que compõem a matriz $X$ descrita em (1) são: desigualdade de renda do período inicial, PIB per capita para o ano 2000 e taxa de crescimento populacional somado a depreciação ${ }^{8}$; também há um conjunto de 14 variáveis de controle para 1486 municípios brasileiros ${ }^{9}$ no ano 2000 as quais são descritas a seguir e que podem compor a matriz $Z$, de acordo com cada cenário formulado. Há variáveis de educação, de finanças públicas, de desenvolvimento financeiro, de saúde, de infraestrutura domiciliar, de abertura comencial, de disposição geográfica, de demografia e proxy's para desenvolvimento. Uma descrição das variáveis utilizadas é exposta no Quadro $1{ }^{10}$

Ademais, para ter uma melhor visualização das variáveis de principal interesse, expõe-se no Gráfico 1, a taxa de crescimento do PIB per capita entre o período de 2000 a 2009 em relação a desigualdade de renda, que é medida pelo coeficiente de Gini do ano 2000, para os municípios brasileiros. Observa-se, pela nuvem de dados, que a priori é difícil estabelecer qual a relação entre desigualdade de renda e crescimento econômico no Brasil.

\footnotetext{
8 Para a taxa de depreciação assume-se o valor de 5\% para todos os municípios, que é o padrão utilizado nas equações empíricas para testes de crescimento.

9 O Brasil possuía 5.507 municípios no ano 2000. Apesar da perda substancial do número de municípios em virtude da carência de informações, os dados foram suficientes para representar a heterogeneidade do país.

${ }^{10}$ Ao final do trabalho é apresentado um apêndice com as estatísticas descritivas destas variáveis.
} 
Gráfico 1: Taxa de crescimento do PIB per capita (2000-2009) e coeficiente de gini (2000) para os municípios brasileiros*.

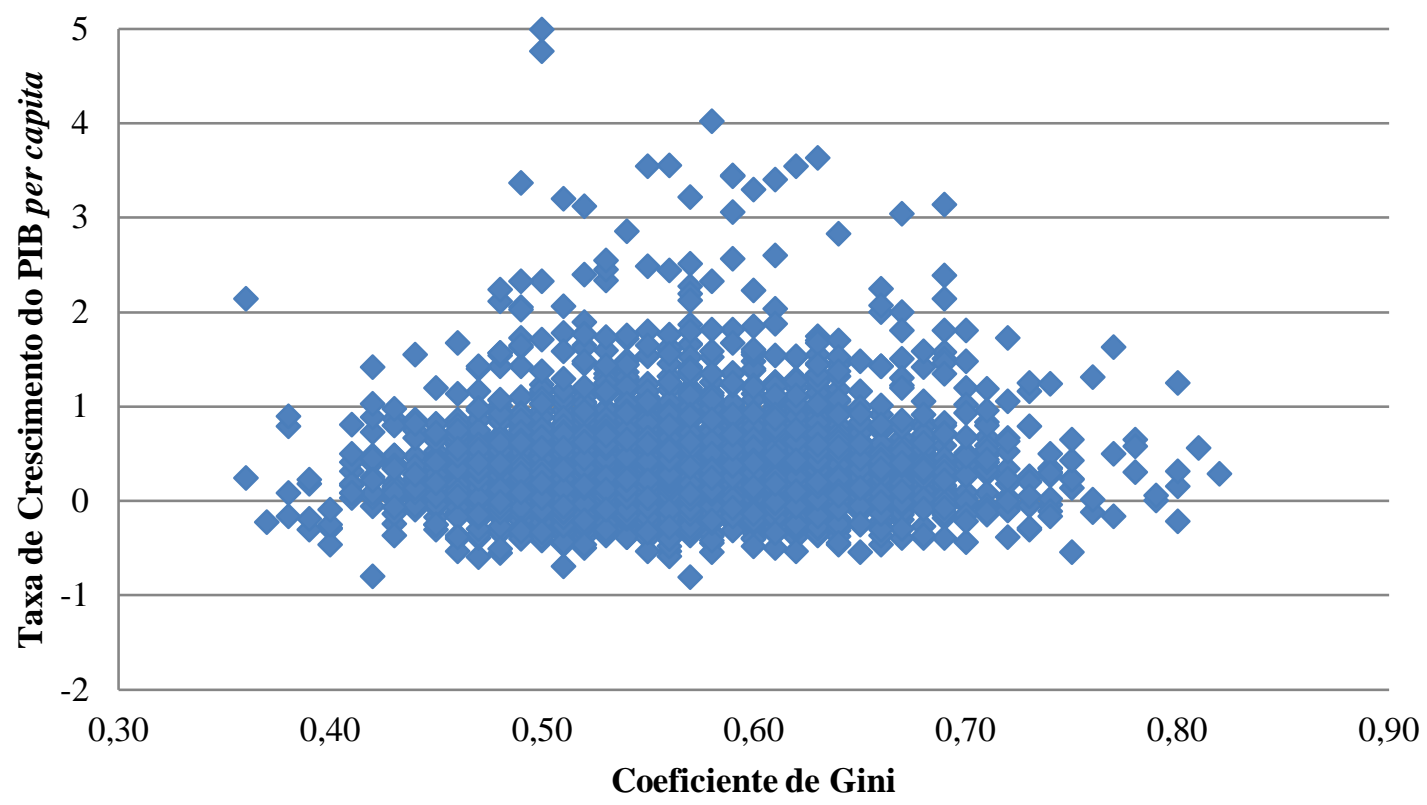

Fonte: IBGE. Elaborado pelos autores.

(*) Excluindo-se os municípios (outliers) que apresentaram taxa decrescimento superior a $10 \%$.

Já a Figura 1, mostra a citada relação de forma espacializada. Quanto mais próxima da cor preta for o municipio pior é sua distribuição de renda e mais baixa é sua taxa de crescimento. Nota-se que os municípios brasileiros caracterizam-se por uma elevada desigualdade de renda e uma baixa taxa de crescimento, com um certo número de exceções.

Figura 1: Mapas com a taxa de crescimento do PIB per capita (2000-2009) e coeficiente de gini (2000) para os municípios brasileiros.
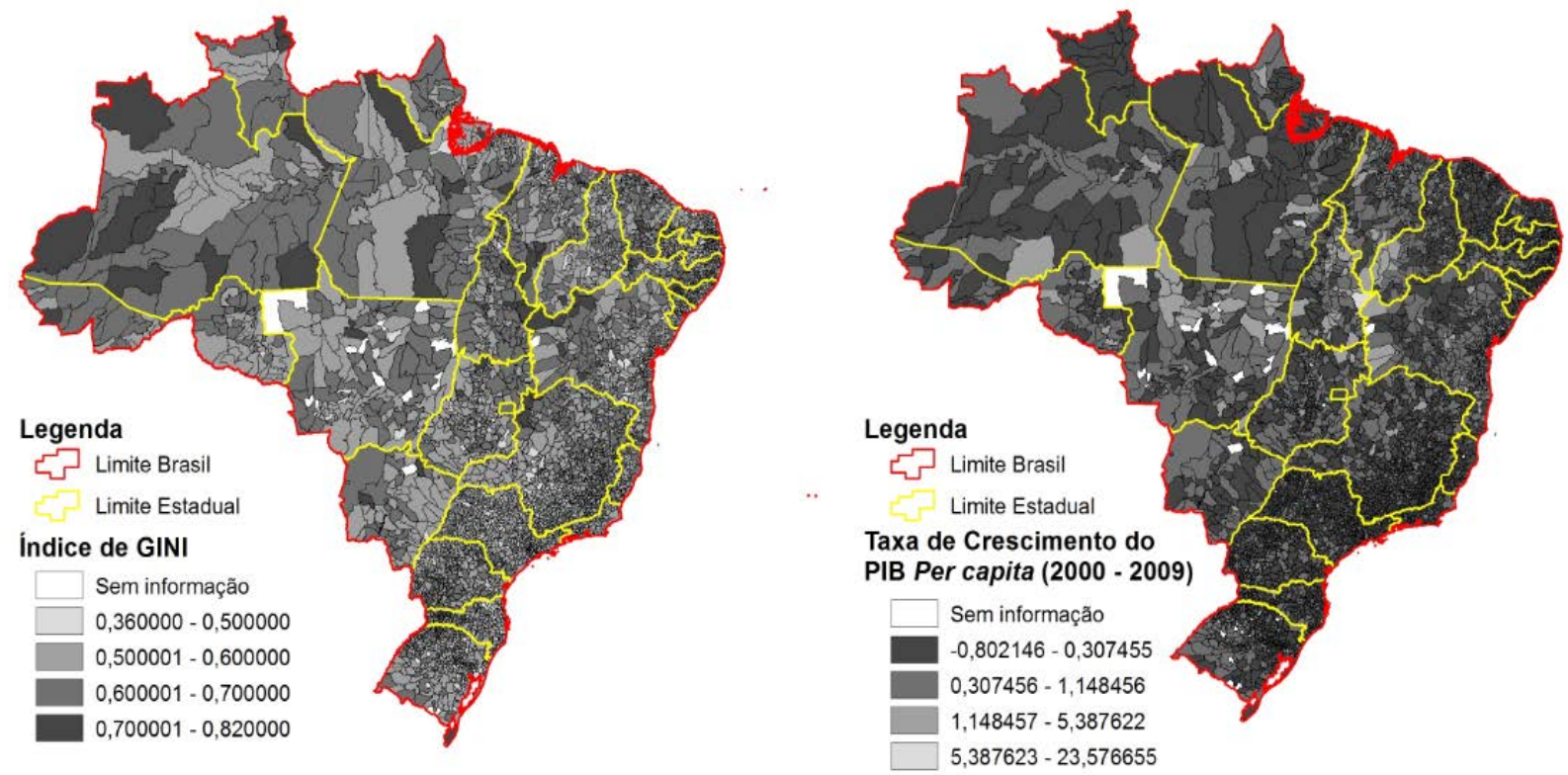

Fonte: IBGE. Elaborado pelos autores. 


\subsection{O Modelo}

A pré-seleção de variáveis de controle realizada em pesquisas empíricas é uma questão importante, pois dificulta um consenso sobre um modelo empírico particular a ser estimado. $\mathrm{O}$ método Model Averaging surge como uma alternativa mais apropriada para se investigar o efeito de uma determinada variável no modelo de regressão. Nesse método, não é necessário optar pelo melhor e único modelo, mas sim levar em consideração, no processo de estimação, diversas configurações desse modelo (Moral-Benito, 2015).

O estudo de Sala-i-Martin et al. (2004) popularizou o uso de Model Averaging como uma ferramenta para pesquisadores que estudam regressões de crescimento, sendo importante ressaltar que existem duas abordagens de Model Averaging: a Frequentist Model Averaging (FMA) e a Bayesian Model Averaging (BMA), que se diferenciam pelo critério de seleção dos pesos do modelo e pelo processo de inferência. Neste trabalho utilizou-se a abordagem Frequentist Model Averaging (FMA), a qual é descrita em maiores detalhes a seguir.

\subsubsection{Model Averaging e Estimador de FMA}

Neste trabalho, a variável dependente, $\Delta Y$, na equação de regressão (1) é taxa de crescimento da economia no período de 2000 a 2009, os regressores mantidos fixos na equação de regressão (1), ou seja, contidos na matriz de regressores $X$, são o coeficiente de Gini, o PIB per capita do peńodo inicial (no caso o ano 2000), e o crescimento populacional mais a taxa de depreciação. As variáveis de controle reunidas em $Z$ são incertas e podem, ou não, serem incluídas no modelo. O vetor de parâmetros $\beta$ deve ser estimado, e o valor de $\beta_{1}$ é que nos prende a atenção, pois, dependendo de seu sinal e de sua significância estatística, é que se pode diagnosticar se a desigualdade tem um efeito positivo, negativo ou nulo sobre o crescimento econômico.

Moral-Benito (2015) assegura que o estimador FMA associado ao vetor de parâmetros $\beta$ pode ser construído da seguinte maneira:

$$
\hat{\beta}_{F M A}=\sum_{j=1}^{2 q} \omega_{M_{j}} \hat{\beta}_{M_{j}}
$$

onde $\omega_{\mathrm{M}_{\mathrm{j}}}$ são pesos atribuídos as estimativas de cada modelo, $0 \leq \omega_{\mathrm{M}_{\mathrm{j}}} \leq 1 ; \sum_{\mathrm{j}=1}^{2^{\mathrm{q}}} \omega_{\mathrm{M}_{\mathrm{j}}}=$ 1.

Assim sendo, estando-se interessado no valor do coeficiente associado à variável de desigualdade, basta que se atenha atenção à $\hat{\beta}_{1 F M A}=\sum_{j=1}^{2 q} \omega_{1 M_{j}} \hat{\beta}_{1 M_{j}}$. Posto isso, é necessário que se compute uma média ponderada de todas as estimativas dos coeficientes das variáveis de controle, que devem ser muitas, não havendo necessidade de optar por um só modelo. Ademais, computam os erros aleatórios associados a cada uma dessas estimativas, o que faz com que seja possível se realizar uma inferência baseada no universo inteiro dos modelos candidatos.

\subsubsection{A Escolha da Ponderação baseada no Critério da Validação Cruzada}

Aqui, seguiu-se a proposição de Hansen e Racine (2012), que consiste em selecionar os pesos do estimador de mínimos quadrados do Model Averaging minimizando um critério de validação cruzada. Essa abordagem ficou conhecida por J ackknife Model Averaging (J MA). Além desta classe de estimadores ser eficiente, uma vez que apresenta erro quadrático médio menor que métodos alternativos, como o Mallows Model Averaging, por exemplo, Hansen e Racine demonstram que ela é mais apropriada para modelos heterocedásticos. 
Segundo os autores, o estimador J MA é escrito da seguinte forma:

$$
\hat{\mu}(\lambda)=\sum_{j=1}^{J} \lambda_{j} \hat{\mu}_{j}
$$

Assim, o vetor de pesos J ackknife é aquele que minimiza a soma dos quadrados dos resíduos da combinação linear dos modelos estimados, isto é,

$$
\begin{gathered}
\hat{\lambda}^{*}=\arg \min C V(\lambda) \\
\lambda \in H_{N}
\end{gathered}
$$

onde $H_{N}=\left\{\lambda \in R / \lambda_{j} \geq o\right.$ e $\left.\sum_{j=1}^{J} \lambda_{j}=1\right\}$.

\subsubsection{Cálculo dos erros padrões de bootstrap}

Para realização do processo de inferência sobre os coeficientes $\hat{\beta}_{F M A}$, adotou-se o cálculo dos erros padrões sugerido por Buckland et al (1997) e Schomaker, Wan e Heumann (2010) ${ }^{11}$. Este procedimento é necessário, pois não se conhece, a priori, a distribuição de probabilidade dos estimadores $\hat{\beta}_{F M A}$. Ademais, essa abordagem conservadora encontra o valor máximo possível para as variâncias que incorporam um componente representado pelo viés de erro de especificação.

A estimação dos erros padrões é realizada da seguinte maneira:

$$
\left.e p(\hat{\beta})=\sum_{j=1}^{K} \omega_{j} \sqrt{\left.e p \overline{\left(\beta^{(J)}\right.}\right)} / v^{i e ́} s_{j}\right)+ \text { viés }^{2} \quad,
$$

onde, $o$ viés $s_{j}=\widehat{\beta^{(1)}}-\widehat{\beta}$ representa o erro de especificação que surge na estimativa $\widehat{\beta}$ sob o modelo j. Como mencionado por Hjort e Claeskens (2003), esta fórmula dá erros padrões razoavelmente mais precisos, os quais incluem a incerteza adicional devido à seleção do modelo.

\section{RESULTADOS E CONSIDERAÇÕES FINAIS}

Conforme foi discutido na revisão de literatura, há uma nítida controvérsia com relação aos efeitos da desigualdade de renda no crescimento econômico, em especial do que trata o caso brasileiro. A pesquisa aqui delineada busca contribuir com a literatura que lida com o tema fazendo uso de uma técnica bastante flexível e ainda pouco difundida no Brasil.

Esta técnica foi proposta em Hansen e Racine (2012) e é conhecida como J ackknife Model Averaging. Tal técnica é uma abordagem Frequentista de Ponderação de Modelos que consiste em estimar todas as possíveis especificações de modelos, dado um amplo conjunto de variáveis, e então calcular um modelo que é uma média ponderada de todos os outros estimados. A escolha dos fatores de ponderação é feita com base na minimização do critério de validação cruzada. Frente às demais, a vantagem dessa técnica é a de que se permite um alto grau de parcimônia ao se determinar a especificação mais coerente para explicar a relação crescimentodesigualdade.

Reuniu-se um conjunto de 17 variáveis, todas elas com algum respaldo teórico, capazes de explicar o crescimento. Devido a limitação computacional, destas 17

\footnotetext{
${ }^{11}$ Ver Amini e Parmeter (2012) para uma discussão detalhada sobre o assunto.
} 
variáveis, 3 foram fixadas para todas as regressões: a desigualdade de renda, medida pelo coeficiente de Gini; o PIB per capita do período inicial e; taxa de crescimento populacional somada a depreciação. Posteriormente, as 14 demais variáveis foram agrupadas dediversas maneiras possíveis, cobrindo combinações de 4, 8 e 12 variáveis. No total, foram estimados 4.095 modelos.

Estes modelos foram ponderados de acordo com o critério de validação cruzada proposto em Hansen e Racine (2012) e formularam-se estatísticas t de Student com base nos erros padrões de bootstrap propostos em Schomaker, Wan e Heumann (2010), os quais também consideram a incerteza adicional devido à seleção do modelo.

Com o intuito de não tornar a análise demasiadamente extensa e como nosso objetivo se concentra única e exclusivamente nos efeitos da desigualdade no crescimento econômico, a análise dos estimadores para as demais variáveis é negligenciada. Assim, nos concentramos apenas nos coeficientes estimados para o Coeficiente de Gini. O valor estimado dos coeficientes, levando em consideração cada combinação de regressores pré-estabelecida, assim como as estatísticas t elaboradas são expostas na tabela a seguir.

Tabela 1- Resultados obtidos pelos estimadores deJMA

\begin{tabular}{cc}
\hline Cenários & $\widehat{\boldsymbol{\beta}}_{J M A}$ \\
\hline$X=x_{1}\left|x_{2}\right| x_{3} ; Z=C_{4}^{14} z_{i}$ & 0,1953 \\
$X=x_{1}\left|x_{2}\right| x_{3} ; Z=C_{8}^{14} z_{i}$ & $(0,5825)$ \\
$X=x_{1}\left|x_{2}\right| x_{3} ; Z=C_{12}^{14} z_{i}$ & 0,0719 \\
& $(0,2606)$ \\
\hline
\end{tabular}

Fonte: Elaborado pelos autores.

A Tabela 1 apresenta as estimativas para o efeito da desigualdade no crescimento econômico com base em três cenários. Todos os cenários mantêm fixos os regressores do índice de Gini, $x_{1}$, do PIB per capita inicial, $x_{2}$, e da taxa de crescimento populacional somada à depreciação, $x_{3}$. No entanto, para que sejam computadas as estimativas do J ackknife Model Averaging, no cenário 1 é feita uma ponderação de todas as combinações possíveis de 4 das 14 demais variáveis; no cenário 2 a ponderação considera todas as combinações possíveis de 8 das 14 demais variáveis; e no cenário 3 consideram-se as combinações de 12 das 14 variáveis explicativas.

Pode-se observar que, para os cenários 1 e 2, há uma relação positiva entre a desigualdade e a taxa de crescimento da economia brasileira no período de 2000 a 2009, corroborando com os estudos desenvolvidos por Li eZou (1998) e, Barro (2000) quando analisado para os países ricos. Já para o cenário 3 se observa uma relação negativa entre as variáveis, o que também foi constatado por Perotti (1996) e Deininger e Squire (1998). Não obstante, ao se considerar as estatísticas t, não se deve rejeitar a hipótese de que os coeficientes estimados são todos iguais à zero, o que implica na ausência de relação entre estas variáveis.

Esse trabalho vem acrescentar a literatura demonstrando que dificilmente será possível encontrar evidências de que a desigualdade de renda exerceu influência sobre 
a taxa de crescimento dos municípios brasileiros no período de 2000 a 2009, pelo menos para o caso em que se utiliza como medida de desigualdade o coeficiente de Gini. Tal resultado contrasta com uma boa parte dos trabalhos anteriores. ${ }^{12}$ Entretanto, e ainda que não sejam diretamente comparáveis, nossos resultados parecem estar de acordo com trabalhos recentes, como o de Teyssier (2013).

Outra questão de interesse é a possibilidade de se incluir efeitos thresholds à técnica de ponderação de modelos. De fato, a ausência de significância estatística associada ao coeficiente de desigualdade pode ser resquícios de um possível efeito não linear influenciando a relação entre estas variáveis. Cruz e Teixeira (2015), por exemplo, reportam algumas evidências para o caso brasileiro nesse sentido. Porém, embora já exista na literatura trabalhos tratando de ponderação de modelos não lineares, ${ }^{13}$ não foi esboçada nenhuma teoria assintótica que comprove a eficácia e valide as estimativas advindas deste método.

Ademais, discutir sobre os efeitos assimétricos da desigualdade de renda sobre o crescimento econômico, possivelmente, esteja mais relacionado com a questão de descobrir as fontes geradoras e propagadoras da desigualdade de distribuição de renda em uma economia e, a partir disso determinar a direção do seu efeito sobre o crescimento.

\section{REFERÊNCIAS BIBLIOGRÁFICAS}

ALESINA, A.; RODRICK, D. Distributive Politics and Economic Growth. Quarterly J ournal of Economics, v.109, p. 465-490, 1994.

ALMEIDA, L. O.; GUILHOTO, J. J. M. 2006. Crescimento Econômico e Distribuição de Renda: Uma análise a partir das estruturas econômicas do Brasil contemporâneo. In: Anais do XXXIV Encontro Nacional de Economia, 2006.

AMINI, S. M.; PARMETER, C. F. Comparison of Model Averaging Techniques: Assessing Growth Determinants. J ournal of Applied of Econometrics, v. 27, p. 870-876, 2012.

AMORIM, A. L.; SILVA. S.P. Desigualdade de renda, pobreza e crescimento econômico nas microrregiões de Minas Gerais: evidência empírica e espacial entre 1980 e 2000. In: Anais do XIV Seminário sobre a economia mineira, 2010.

BANERJ EE, A. e DUFLO, E. Inequality and growth: What can the data say? J ournal of Economic Growth, v. 8, p. 267-299, 2003.

BARRETO, F. A. F. D.; J ORGE NETO, P. M.; TEBALDI, E. Desigualdade de renda e crescimento econômico no Nordeste brasileiro. Revista Econômica do Nordeste, v. 32, p. 842-859, 2001. Número Especial.

\footnotetext{
12 Acreditamos quea falta de consenso nos resultados encontrados pela literatura ocorre, possivelmente, devido às especificações errôneas dos modelos e, sem desmerecer o esforço de pesquisa, que podem apresentar o que Roodman (2007) define como publication bias, ou seja, a busca por resultados estatisticamente significantes, por serem de mais fácil publicação.

13 Ver Shen, Zhang, Barth (2004) e Shen, Zhang, Barth e Goldfarb (2006).
} 
BARRO, R.J . Economic Growth in a Cross Section of Countries. The Quarterly J ournal of Economics, v. 106, p. 407-443, 1991.

BARRO, R. J . Inequality and growth in a panel of countries. J ournal of Economic Growth. 5(1), p.87-120, 2000.

BARRO, R.; X. SALA-I-MARTIN. Public Finance in Models of Economic Growth. Review of Economic Studies, v. 59, p. 645-662, 1992.

BARROS, R. P. de; HENRIQUES, R.; MENDONÇA, R. Desigualdade e pobreza no Brasil: retrato de uma estabilidade inaceitável. Revista Brasileira de Ciências Sociais, v.15, n. 42, 2000.

BOURGUIGNON, F. e VERDIER, T. Oligarchy, Democracy, Inequality and Growth. J ournal of Development Economics, v.62, p. 285-313, 2000.

BUCKLAND, S. T.; BURNHAM, K. P.; AUGUSTIN. N. H. Model Selection: An Integral Part of Inference. Biometrics, Vol. 53, No. 2, J un, p. 603-618, 1997.

CASTRO, R. S. PORTOJ R, S.S. Efeitos da desigualdade de renda sobre o crescimento econômico no Brasil: uma analise não linear. Perspectiva Econômica, v.3, n. 1, p. $27-61,2007$.

CRUZ, P. B.; TEIXEIRA, A. C. C.; MONTE-MOR, D. S. O efeito da desigualdade da distribuição de renda no crescimento econômico. Revista Brasileira de Economia, v. 69, n. 2, p. 163- 186, 2015.

DATT, G. RAVALLION, M. Growth and redistribution components of changes in poverty measure: A decomposition with application to Brazil and India in the 1980s. J ournal of Development Economics, Vol.38, pp. 275-295, 1992.

DEININGER, K. e L. SQUIRE. New Ways of Looking at Old Issues: Inequality and Growth. J ournal of Development Economics, 57, p. 259-287, 1998.

DURLAUF, S. N.; QUAH, D. T. The New Empirics of Economic Growth. In: Taylor, J . and Woodford, M. Chapter 04 in Handbook of Macroeconomics, vol. 1, Part A, pp. 235-308, Elsevier, 1999.

DURLAUF, S. N., JONHSON, P. A.; TEMPLE, J. R. W. Growth econometrics. In P. Aghion and S. N. Durlauf (eds.) Handbook of Economic Growth, Volume 1A, North-Holland: Amsterdam, 2005, pp. 555-677, 2005.

FORBES, K.J . A Reassessment of the Relationship between Inequality and Growth. American Economic Review, 90, pp. 869-887, 2000.

GALOR, O. E ZEIRA, J. Income distribution and macroeconomics. Review of Economic Studies, v. 60, p. 35-52, 2003.

HANSEN, B. E.; Least Squares Model Averaging. Econometrica, vol. 75, n. 4, pp. 1175-1189, J uly, 2007.

HANSEN, B. E.; RACINE, J. S. Jackknife Model Averaging. Journal of Econometrics, vol. 167, pp. 38-46, 2012. 
HJ ORT, A. R.; CLAENSKENS, G. Frequentist Model Average Estimators. J ournal of the American Statistical Association, vol. 98, pp. 879-899, 2003.

KUZNETS, S. Economic growth and income inequality. American Economic Review. v. 45, p.1-28,1955.

LI, H. e ZOU, H. Income Inequality is not Harmful for Growth: Theory and Evidence. Review of Development Economics, 2, pp. 318-334, 1998.

LIANG, H.; ZOU, G.; WAN, A.T.K.; ZHANG, X. Optimal weight choice for Frequentist Model Average Estimators. Journal of the American Statistical Association, vol. 106, pp. 1053-1065, 2011.

LOPES (2004). Pro-Poor-Growth: Is there a Trade Off? The World Bank, Policy Research Working Paper No.3378.

OLIVEIRA, L. G.; NERI, M. C. Prometeu Acorrentado? Um Estudo Empírico dos Efeitos da Desigualdade sobre o Crescimento. 2009, p.36. Dissertação (Mestrado Acadêmico em Economia). Escola de Pós-Graduação em Economia, Fundação Getúlio Vargas.

ORTEGA-DIAZ, A. Assessment of the Relationship between Income Inequality and Economic Growth: A Panel Data Analysis of the 32 Federal Entities of Mexico, 1960-2002, in Badi H. Baltagi (ed.) Panel Data Econometrics Theoretical Contributions and Empirical Applications (Contributions to Economic Analysis, Volume 274) Emerald Group Publishing Limited, pp.361 - 381, 2006.

MANKIW, N. G.; ROMER, D.; WEIL, D. N. A contribution to the empirics of economic growth. Quarterly J ournal of Economics, n. 107, pp. 407-437, 1992.

MENDES, M. J. Desigualdade e Crescimento: uma revisão da literatura. Brasília: Núcleo de Estudos e Pesquisas/CONLEG/Senado, ago/2013 ( Texto para Discussão no 131).

MORAL-BENITO, Enrique. Model averaging in economics: An overview. J ournal of Economic Surveys, v. 29, n. 1, p. 46-75, 2015.

PEROTTI, R. Growth, Income Distribution and, Democracy: What can the Data Say. J ournal of Economic Growth, 1, pp. 149-187, 1996.

PERSSON, T. e G. TABELLINI. Is Inequality Harmful for Growth? American Economic Review, 84, pp. 600-621, 1994.

RAO, P.; MILLER, R. L. Applied Econometrics. Hardcover, 1971.

RODRICK, D. Where did all the growth go? External shocks, social conflict, and growth collapses. Harvard University, mimeo, 1998.

ROODMAN, D. A Short Note on the Theme of Too Many Instruments. Working Paper. 125, Center for Global Development. Washington, DC. 2007. 
SALA-I-MARTÍN, X., G. DOPPELHOFER, and R. MILLER. Determinants of LongTerm Growth: A Bayesian Averaging of Classical Estimates (BACE) Approach, American Economic Review, 94, 813-835, 2004.

SCHOMAKER, M.; WAN, A T. K.; HEUMANN, C. Frequentist Model Averaging with missing observations. Computation Statistics \& Data Analysis, vol. 54, pp. 33363347, 2010.

SHEN, X.; ZHANG, J .; BARTH, E. J .; GOLDFARB, M. Nonlinear Model-Based Control of Pulse Width Modulated Pneumatic Servo Systems. J ournal Dynamic Systems, Measurement, and Control, vol. 128, pp. 663-669, 2006.

TEYSSIER, G. Inequality, inequality of opportunity, and growth: what are we talking about? Theory and empirical investigation in Brazil. Université Paris 1Panthéon Sorbonne. Version 1- 2, Dec, 2013. 


\section{APÊNDICE}

A Tabela 2, a seguir, apresenta uma análise descritiva das variáveis utilizadas, pode-se observar que a taxa de crescimento do PIB per capita, no período de 2000 a 2009, nos 1486 municípios brasileiros oscilou no intervalo de -0,54 a 5,00. A média do Gini no período inicial era 0,55 e, destaca-se a alta taxa de analfabetismo, com municípios possuindo, aproximadamente, $60 \%$ da sua população sem saber ler e escrever um bilhete simples.

Tabela 2 - Medidas-resumo das variáveis utilizadas nas regressões.

\begin{tabular}{lrrrr}
\hline Variável & Média & $\begin{array}{r}\text { Desvio } \\
\text { Padrão }\end{array}$ & Mínimo & Máximo \\
\hline$\Delta$ Y & 0,27 & 0,43 & $-0,54$ & 5,00 \\
Gini & 0,55 & 0,05 & 0,38 & 0,80 \\
PIBpc & 6.422 & 5.756 & 808 & 96.759 \\
$\mathbf{N}$ & 0,15 & 0,19 & $-0,424$ & 2,33 \\
Exp/Pib & 1,87 & 3,45 & 0,00 & 35 \\
Agr/tot & 0,15 & 0,14 & 0,00 & 0,95 \\
Gov/Pib & 0,08 & 0,04 & 0,01 & 0,34 \\
Rur/pop & 0,24 & 0,19 & 0,00 & 0,92 \\
Água & 85,55 & 19,10 & 6,32 & 99,98 \\
Energia & 94,94 & 9,31 & 39 & 100 \\
Mort & 24,18 & 12,95 & 5,38 & 83,11 \\
Esp & 70,29 & 3,73 & 55,41 & 78,18 \\
Analf & 16,47 & 10,35 & 2,02 & 59,98 \\
MAE & 5,11 & 1,11 & 1,64 & 9,65 \\
Po & 41,68 & 7,82 & 15,76 & 68,87 \\
Distcap & 223,17 & 160,82 & 0,00 & 819,92 \\
Dfo & 0,11 & 0,14 & 0,00 & 2,29 \\
Dfd & 3,02 & 95,81 & 0,00 & 3735 \\
\hline
\end{tabular}

Fonte: IBGE. PNUD. Elaborado pelos autores. 